\title{
A new approach to the design and construction of the nearly-zero-energy building in Sardinia
}

\author{
M. Basciu ${ }^{1,2}$ \\ ${ }^{1}$ Department of Civil Engineering, Environmental Engineering and \\ Architecture, University of Cagliari, Italy \\ ${ }^{2}$ Network of Sardinian companies "Domu Noa”, Italy
}

\begin{abstract}
International commitments have led the $\mathrm{EU}$ to the adoption of increasingly strict regulations on the reduction of energy consumptions: currently, the benchmark for quality construction is the nearly-zero-energy building. Consequently, Italian governments have implemented a continuous regulatory update. This radical change, in the Sardinian context, traditionally characterized by low sensitivity to these issues, has substantially modified the constructions market. The professionals in the construction field have developed a new attention to energy efficiency, but the current objectives require additional investments in innovation.

A holistic approach to the design and construction of buildings that implies a general renewal, is therefore essential. Designers and contractors will have to be able to provide a quality product at competitive prices and the building characteristics will not have to result from the adaptation of models developed in other cultures and climates, but from the local industry experience and from the local research.

The improvement of the product will encourage cultural growth of building industries and will enhance public information triggering a virtuous circle that stimulates the diffusion of energy-saving culture.

In collaboration with the network of Sardinian companies "Domu Noa", research about the issues affecting the design and construction of efficient buildings is being developed. In particular, the aim of this paper is the analysis of the construction systems used by "Domu Noa", the identification of the criticalities of these systems and the definition of constructive solutions.

The general objective is to compose a document that consists of a set of rules and technical solutions for the design and construction of efficient buildings.
\end{abstract}


Using this tool it could be possible to overcome the traditional way to design, which does not guarantee a constant quality of the products, in order to reach the acquisition of methodological systems for the Sardinian context.

Keywords: energy efficiency, passive design, sustainable buildings, building elements.

\section{Introduction}

The enactment of the Energy Efficiency Directive 2010/31/EU [1] urged the Member States to make more decisive policies concerning the existing buildings and new ones. Consequently the Italian governments have implemented a continuous regulatory update.

The introduction of the concept of the nearly-zero-energy building, a building that has a very high energetic performance, so that the very low amount of energy required should be covered to a very significant extent by energy from renewable sources, is particularly interesting. The design and construction of a building with similar performance requires the combination of several factors: the correct thickness of the thermal insulation, the proper execution of connections (no thermal bridges), a careful design of the form, the exposure and the internal arrangement, the integration of high-efficiency plant systems with renewable energy sources. A holistic approach to the design and construction of buildings, is therefore essential. That implies a general renewal of designers and especially contractors, which will have to be able to provide a quality product at competitive prices: the building characteristics will not have to result from the adaptation of models developed in other cultures and climates, but from the local industry experience and from the local research.

This radical change, in the Sardinian context, traditionally characterized by low sensitivity to these issues, has substantially modified the constructions market.

In recent years the professionals in the construction field have developed a new attention to energy efficiency trying to experiment new systems and to coordinate with each other, but the current EU objectives require additional investments in innovation.

The research project "Innovative approaches to the construction process of energy efficient buildings to increase the competitiveness of Sardinian companies" developed in collaboration with the network of three Sardinian companies "Domu Noa", through a research grant financed from the resources of R.O.P. SARDINIA E.S.F. 2007-2013 - Objective Regional competitiveness and employment, Priority IV Human Capital, Lines of Activities 1.1.1. and 1.3.1", belong to this field.

The work, concerning the issues affecting the efficient buildings and the collaboration between the three companies, is about design of the building-plant system, the definition of efficient envelope and the integration with different kind of plant systems, the analysis of construction details.

In particular the attention is focused on the use of innovative envelopes and on the difficulties on realization of the connections between the building elements (for example the thermal bridge between the wall and the window) and of the integration with plant systems. 
The analyses and studies conducted in the early months of work proposed some solutions, which aim to solve the main problems related to the winter behavior of envelopes made by polystyrene.

Currently, the research, is examining the summer behavior of buildings made by construction systems used by "Domu Noa", in order to guarantee not only the reduction of heating and cooling demand, and the absence of constructive deficits, but also to guarantee the comfort inside the building during the summer.

The main objective is to compose a document that consists of a set of rules and technical solutions for the design and construction of efficient buildings and for the energy retrofit. Using this tool it could be possible to overcome the traditional way to design, which does not guarantee a constant quality of the products, in order to reach the acquisition of methodological systems for the construction of energy efficient building in the Sardinia.

This document will be integrated with the drafting of a user manual, which explains to the inhabitants in simple way the rules to follow to enhance the performances of these buildings characterized from the envelope well insulated and increasingly airtight.

\section{The case study}

\subsection{A new envelope for nearly-zero-energy building}

The introduction of the concept of the nearly-zero-energy building, has totally changed the way to work of building companies that will have to optimize the construction process in the phase of design, construction and user information. Within the biennium 2018-2020, indeed, the companies will have to be able to provide a high energetic quality product at reasonable cost.

For this reason, the network of companies Domu Noa has begun the testing of applications, in Sardinian climate, of innovative envelopes that would guarantee high energy efficiency and ease of installation.

Currently, a system based on modular sheets of polystyrene with steel welded wire mesh, completed with the application of the concrete, is being analyzed. These sheets could be used both for load-bearing function and for non-load bearing function, for the realization of the envelope (walls and slabs) and other building elements such as the stairs. The thickness of the polystyrene may vary according to the thermal performance required and, if it is necessary, the element of the envelope can be completed with the integration of layers of different materials to improve, for example, the acoustic performances.

The use in construction of buildings (or renovation of existing buildings) of multilayer components with polystyrene core, if placed in a building process that from design to construction, is thought to exploit the characteristics of the product, should make companies more competitive because of the reduction of costs and times to realization. 


\subsection{The energy consumptions of the building}

The first realization with construction system with polystyrene core dates back to 2012. It is a building, with reinforced concrete frame, located on the outskirts of Uta (1070 degree days), a small town in the province of Cagliari. The building has three floors (ground floor, first floor, second floor) and is divided into seven apartments.

Because of the NW-SE exposure and the layout not all apartments have a good orientation. As it is shown in figure 4, the ground floor and the second floor are divided into two apartments, one mainly oriented to the south and one mainly oriented to the north, the first floor is divided into three apartments, one mainly oriented to the south, one to the north and one exclusively oriented to north-west.
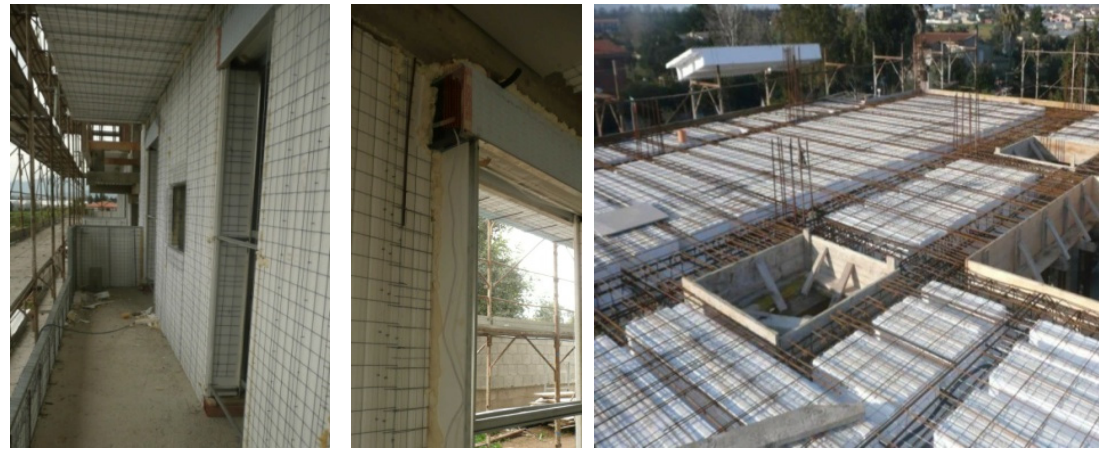

Figure 1: The construction system.

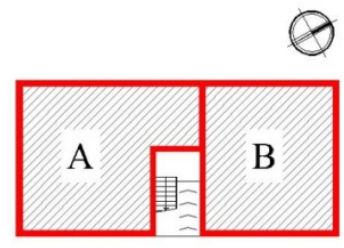

ground floor

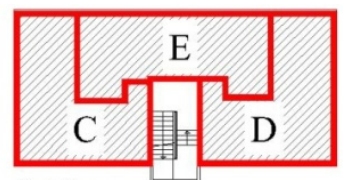

first floor

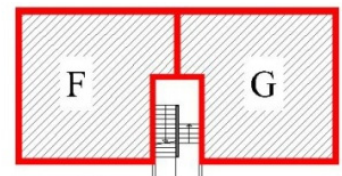

second floor

Figure 2: The arrangement of apartments for each floor.

The walls are composed of an external layer of polystyrene sheet $(12 \mathrm{~cm})$ coated on both sides with a layer of concrete $(2.5 \mathrm{~cm})$ reinforced with steel mesh and by an inner layer of high-density rock wool $(5 \mathrm{~cm})$ and plasterboard $(2.5 \mathrm{~cm})$. The external and inner layer are divided by an air cavity $(8 \mathrm{~cm})$.

The consequent performances are a thermal transmittance of $0.23 \mathrm{~W} / \mathrm{mqK}$ and a periodic thermal transmittance of $0.05 \mathrm{~W} / \mathrm{mqK}$.

Similarly the floor slabs are composed of a slab of reinforced concrete and polystyrene of variable thickness, from $28 \mathrm{~cm}$ to $30 \mathrm{~cm}$, coated on the underside with a layer of concrete $(2.5 \mathrm{~cm})$ reinforced with steel mesh. The floor is 
completed by waterproofing and tiles, in the roof, floating screed and tiles, for the floors.

The performances of the roof are:

- Thermal transmittance $0.27 \mathrm{~W} / \mathrm{mqK}$ and periodic thermal transmittance $0.2 \mathrm{~W} / \mathrm{mqK}$.

The performances of the floors are:

- Thermal transmittance $0.28 \mathrm{~W} / \mathrm{mqK}$ and periodic thermal transmittance $0.12 \mathrm{~W} / \mathrm{mqK}$.

The ground floor was made with an uninsulated crawl space.

The windows, characterized by a wooden frame and a double glazing with low emissivity, have, on average, a transmittance of $1.7 \mathrm{~W} / \mathrm{mqK}$.
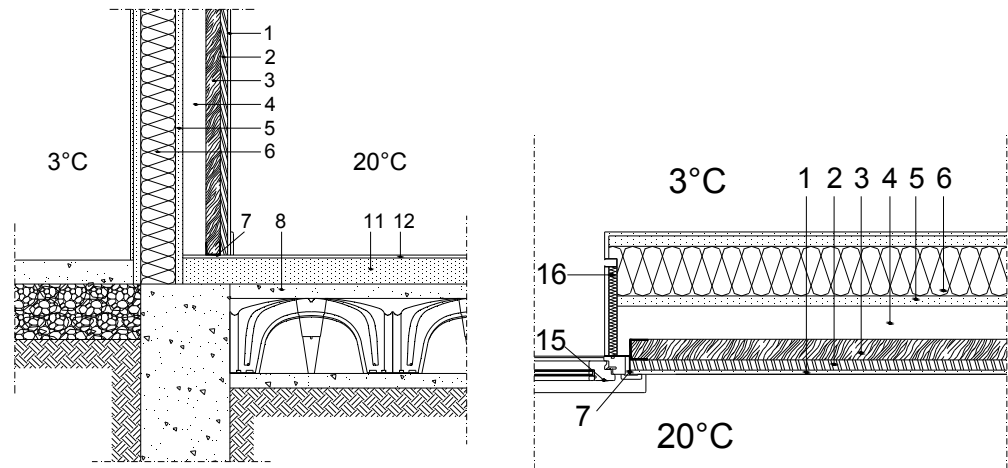

Figure 3: Ground slab and wall (on the left) and wall and window (on the right).

\begin{tabular}{|c|c|c|}
\hline \multicolumn{3}{|l|}{ Legend (fig. 3, 4, 6). } \\
\hline 1. Plaster & 6. Polystyrene sheet $(12 \mathrm{~cm})$ & 12. Tiles \\
\hline 2. Plasterboard $(2.5 \mathrm{~cm})$ & 7. Aluminum rail & 13. Polystyrene \\
\hline 3. High-density rock wool $(5 \mathrm{~cm})$ & 8. Reinforced concrete & $(23 \mathrm{~cm}$ to $25 \mathrm{~cm})$ \\
\hline 4. Air cavity $(8 \mathrm{~cm})$ & 9. Polystyrene sheet $(3 \mathrm{~cm})$ & 14. Polystyrene $(5 \mathrm{~cm})$ \\
\hline 5. Concrete $(2.5 \mathrm{~cm})$ reinforced & 10.Acoustic insulation sheet & 15. Window \\
\hline with steel mesh & 11. Floating screed $(9 \mathrm{~cm})$ & 16. Polystyrene \\
\hline & & $\begin{array}{l}\text { 17. Concrete column } \\
\text { 18. OSB }\end{array}$ \\
\hline
\end{tabular}

The heating and cooling system chosen is an air-air heat pump (COP 3.38) and the hot water is produced by a solar heating system integrated by a gas boiler.

The air changes are guaranteed by natural ventilation.

As it is shown in Table 1, the good level of insulation assures, in the most cases, low winter heat losses (Epi,env) and heating consumption (Epi) well below the values prescribed by law (Epi lim). These indicators and the consumptions for the production of hot water (Epacs), are used for assess the total primary energy demand (Epgl), obtained by adding Epi with Epacs and, consequently, the energy class: four apartments are inscribed in the energy class A, while three in the energy class B. The energy indicators are calculated by the method of the UNI/TS 11300-1-2-4:2008 [2-4]. 

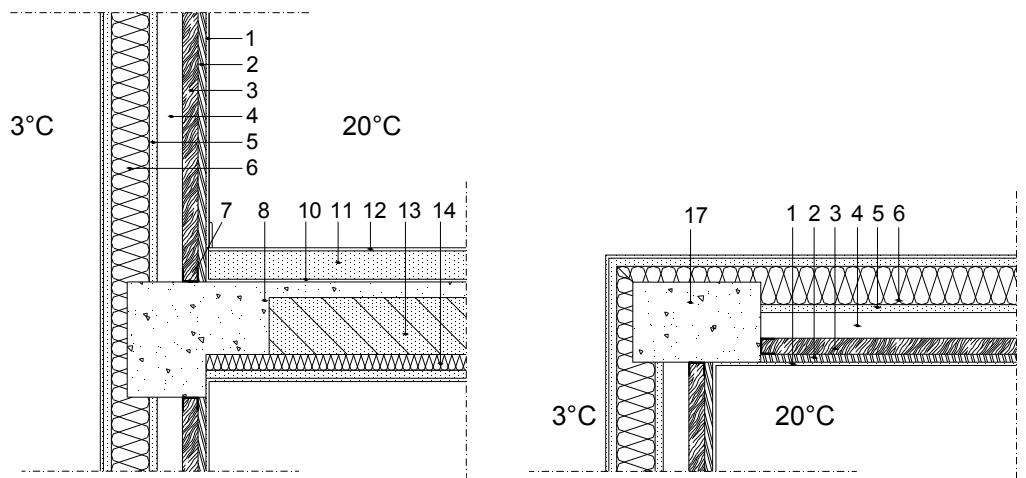

Figure 4: Wall and floor (on the left) and column at corner (on the right).

The two apartments located on the ground floor (A apartment and B apartment) and the apartment located on the first floor and exposed exclusively to north-west (E apartment) belong to class B.

The winter performance of the apartments on the ground floor is due to the lack of insulation in the layers of the ground slab. The use of a sheet of polystyrene $(3 \mathrm{~cm})$ would have been sufficient to reduce consumption winter to reach class A.

With regard to the apartment of the first floor (E apartment), the energy class $\mathrm{B}$ is due, in part, to the consumption for hot water, that in small apartments are very high compared to the demand for heating, in part due to the incorrect exposure, that also compromises the summer performances. Indeed carrying out an initial assessment of the summer behavior of the envelope (Epe, env), by the method of the UNI/TS 11300-1:2008, while the other apartments are in class II (good behavior), this apartment is inserted in class III (poor behavior).

Table 1: Energy consumptions for each apartment.

\begin{tabular}{|c|c|c|c|c|c|c|c|c|c|}
\hline & & & & ter per & ormanc & & & Summer $\mathrm{p}$ & \\
\hline \multirow{2}{*}{ Apart. } & \multirow{2}{*}{$\begin{array}{l}\text { form } \\
\text { factor }\end{array}$} & Epi max & Epi, env & Epi & Epacs & Epgl & \multirow{2}{*}{ class } & Epe, env & \multirow{2}{*}{ class } \\
\hline & & \multicolumn{5}{|c|}{$\mathrm{kWh} / \mathrm{mq}$ year } & & $\mathrm{kWh} / \mathrm{mq}$ year & \\
\hline A & 0.67 & 41.87 & 30.43 & 15.62 & 15.08 & 30.70 & B & 13.80 & II \\
\hline B & 0.66 & 41.27 & 31.65 & 16.23 & 15.27 & 31.51 & B & 17.03 & II \\
\hline $\mathrm{C}$ & 0.48 & 31.42 & 13.50 & 6.94 & 16.27 & 23.21 & A & 17.59 & II \\
\hline D & 0.47 & 30.96 & 14.80 & 7.61 & 16.26 & 23.88 & A & 19.45 & II \\
\hline E & 0.26 & 19.29 & 10.08 & 5.38 & 16.27 & 21.65 & B & 28.00 & III \\
\hline F & 0.70 & 43.77 & 19.60 & 10.09 & 15.47 & 25.56 & A & 17.55 & II \\
\hline G & 0.70 & 43.82 & 21.43 & 11.01 & 15.48 & 26.49 & A & 19.15 & II \\
\hline
\end{tabular}




\section{The analyses in the early months}

The analyses and studies conducted in the early months of work proposed some solutions, which aim to solve the main problems related to the winter behavior of envelopes made by polystyrene.

Indeed, the first applications of modular construction system based on polystyrene sheets have highlighted the difficulties associated with the importation of the systems studied in other climates and other cultural contexts. It often happens that highly efficient and innovative envelopes are assembled with systems established by the local traditions of construction and developed for building systems totally different.

The construction system was chosen because it was supposed to ensure speed of installation, constant quality, high energy performances and low building costs. However, during the first two years of use of the building made by this system, defects of the envelope have emerged. In particular in some apartment occurred the formation of mould in the connections between building components.

Therefore, an accurate investigation, launched during the first months of the work and shown in a previous paper (about to be published), led to the identification of the causes and the solutions of this phenomenon. These deficits are partly due to errors in applications of the construction system (particularly the thermal bridges), in part to the wrong design, as the bad orientation or, especially, to the association of this type of hermetic envelope to the natural ventilation handled in a nonconscious way by the users.

As an example of the work made, in figures 5 and 6 the analysis of thermal bridge between wall and window (detail in figure 3) has been summarized. The thermal bridges are calculated by the method of the UNI EN ISO 10211:2008 [6].
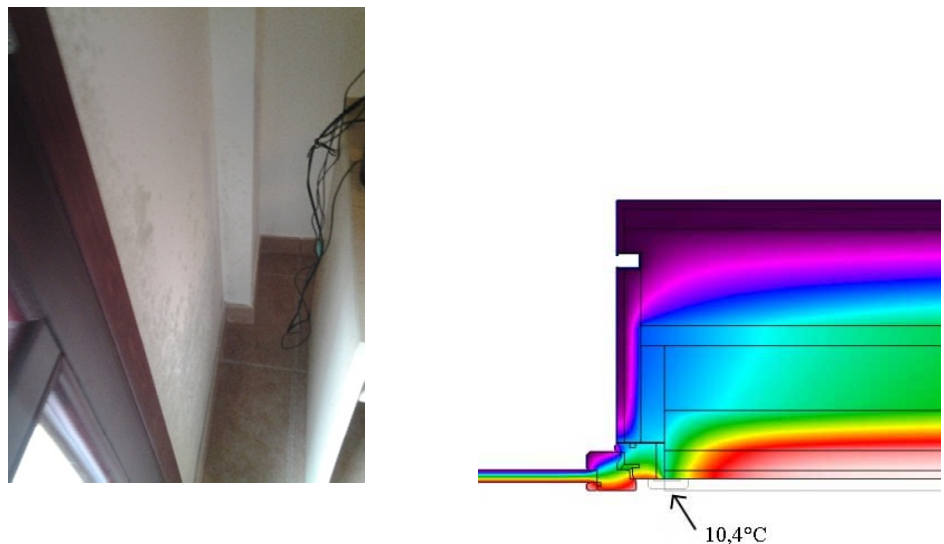

Figure 5: The thermal bridges between wall and window: the mould (on the left) and the temperature trend (on the right) 

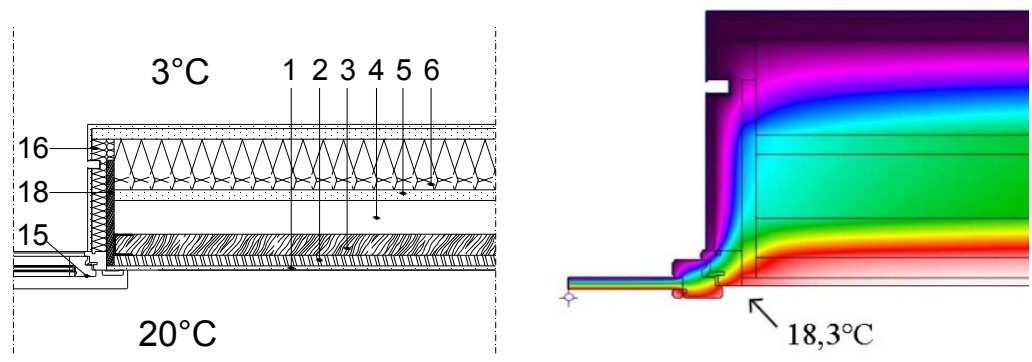

Figure 6: The proposed solution of the thermal bridge between wall and window (on the left) and its new temperature trend (on the right).

\section{The summer behaviour}

The analyses of the construction are continuing deepening the speech on the behavior during the summer. This system, in fact, was developed in colder climates than in Sardinia, where the problem of overheating is less pressing.

The layers used for the walls, consisting of polystyrene sheets integrated with plasterboard and rock wool, ensure good performance both in terms of thermal transmittance (U) and of periodic thermal transmittance (Yie), while the internal areal heat capacity (k1) is low. From the first analysis carried out in dynamic mode (UNI EN ISO 13786:2008 [6]) by evaluating the perceived temperature in rooms without air-conditioning (fig. 7), it is clear that the building, in the summer season, does not guarantee the well-being of the users.

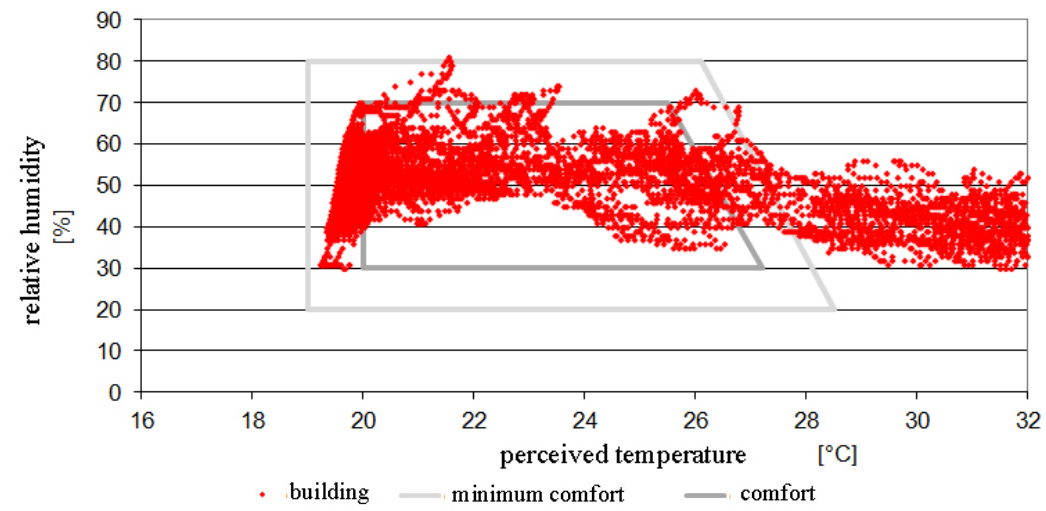

Figure 7: The analysis of the well-being of the users in B apartment.

This phase of work consists in the development of strategies that improve the inner summer conditions. The benefits obtained by the introduction of alternative envelope layers and by the introduction of passive cooling systems (e.g. the natural night ventilation [7]), are being evaluated. In this way it is possible to select actions 
finalized to the optimization of building performances during the summer, reducing the need to use air-conditioning systems.

First, evaluations on the benefits obtained from increased areal heat capacity were made. This parameter represents the actual capacity of the heat accumulation on the inner side of the building elements and is therefore essential for the control of the energy due to internal loads and, consequently, for the attenuation of the operative temperature [8].

We then proceeded to the simulation of the dynamic behavior of the apartment B, in the hottest week of the year (from 22nd July to 29th July), by comparing the operative temperatures obtained using the envelope in polystyrene sheets integrated with plaster board and rock wool (envelope 1) and a massive envelope (envelope 2) composed of solid bricks $(25 \mathrm{~cm})$ and exterior insulation $(13 \mathrm{~cm})$. As can be seen from table 2 the thermal characteristics of the two envelopes differ only in the areal heat capacity.

Table 2: $\quad$ Thermal characteristic of envelope 1 and envelope 2.

\begin{tabular}{|c|c|c|c|}
\hline & $\mathrm{U}$ & Yie & $\mathrm{k} 1$ \\
\hline & $\mathrm{W} / \mathrm{mqK}$ & $\mathrm{W} / \mathrm{mqK}$ & $\mathrm{kJ} / \mathrm{mqK}$ \\
\hline Env.1 & 0,23 & 0,04 & 39,6 \\
\hline Env.2 & 0,23 & 0,03 & 64,8 \\
\hline
\end{tabular}

The results obtained (fig. 8 and fig. 9) show a difference in maximum operative temperature up to almost a degree: the temperature observed using the envelope 1 is $35.6^{\circ} \mathrm{C}$ and the temperature observed with the envelope 2 is $34.9^{\circ} \mathrm{C}$. This improvement is unsatisfactory since the maximum tolerable temperature is equal to $28^{\circ} \mathrm{C}$.

The low values of periodic thermal transmittance, reducing the external loads, determine the consequent reduction in internal overheating and therefore the impact of the areal heat capacity on the internal operative temperature.

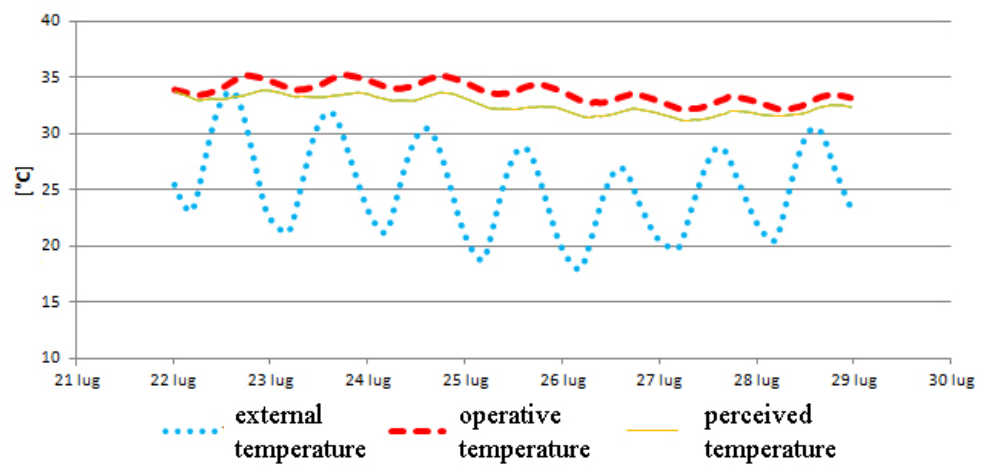

Figure 8: The operative temperature in the case of use of the envelope 1. 


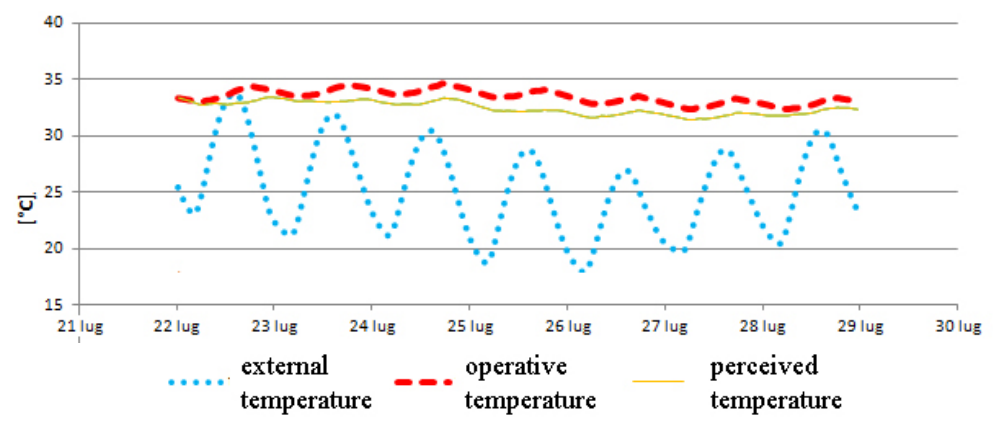

Figure 9: The operative temperature in the case of use of the envelope 2.

The exclusive increased of the areal heat capacity is therefore not the right strategy to improve the inner comfort of a building with these characteristics of thermal transmittance and periodic thermal transmittance, but it must be combined with the use of other systems.

Then the benefits obtained by the introduction of the natural night ventilation, in the case of use of the envelope 1, are been evaluated. As it is shown in figure 10 , the maximum operative temperature is reduced at a value of $30.1^{\circ} \mathrm{C}$.

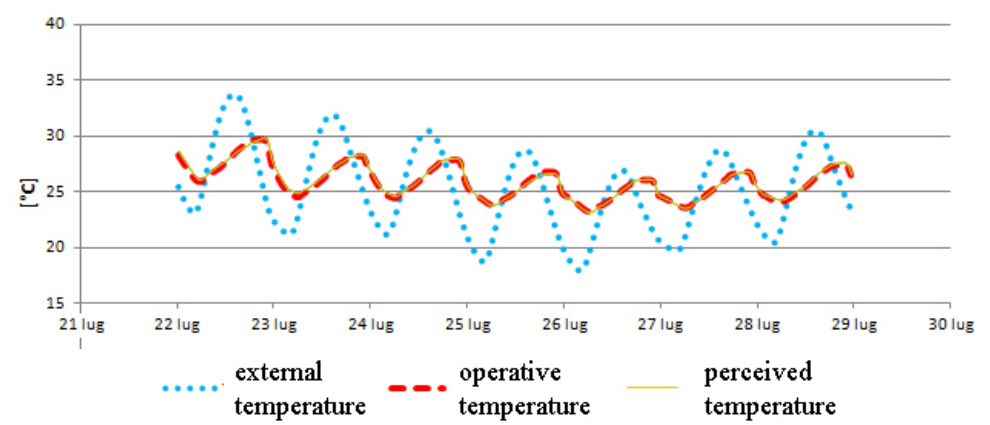

Figure 10: The operative temperature in the case of use of the envelope 1 combined with the natural night ventilation.

At this point, in order to optimize the behavior of the building, the use of night ventilation combined with an envelope characterized by a greater mass on the inner side, has been evaluated.

In particular we have studied two ways to change the envelope 1, the first involves the replacement of the rock wool with wood wool cement, the second involves the replacement of plasterboard with a plasterboard containing microcapsules of PCM [9] (PCM Knauf Smart-Board), obtaining the following layers:

- $\quad$ The envelope 3, composed of an external layer of polystyrene sheet (12 $\mathrm{cm})$ coated on both sides with a layer of concrete $(2.5 \mathrm{~cm})$ reinforced with steel mesh and by an inner layer of wood wool cement $(8 \mathrm{~cm})$ and 
plasterboard $(2.5 \mathrm{~cm})$. The external and inner layer are divided by an air cavity $(5 \mathrm{~cm})$.

- The envelope 4, composed of an external layer of polystyrene sheet $(12 \mathrm{~cm})$ coated on both sides with a layer of concrete $(2.5 \mathrm{~cm})$ reinforced with steel mesh and by an inner layer of high-density rock wool $(5 \mathrm{~cm})$ and Knauf PCM Smart-Board $(2.5 \mathrm{~cm})$. The external and inner layers are divided by an air cavity $(8 \mathrm{~cm})$.

The behavior of envelope 4 has been evaluated considering that the heat capacity of a wall, equipped with a double layer of $15 \mathrm{~mm}$ PCM SmartBoard, is comparable to a $14 \mathrm{~cm}$ thick concrete wall.

The improvement of the operative temperature obtained using the envelope 3 is negligible, whereas the introduction of plasterboard with PCM reduces the maximum temperature at the value of $29.6^{\circ} \mathrm{C}$ (fig. 11).

Moreover, as it is shown in figure 12, during the summer season, the temperature of $28^{\circ} \mathrm{C}$ is exceeded few times. Therefore the simultaneous use of

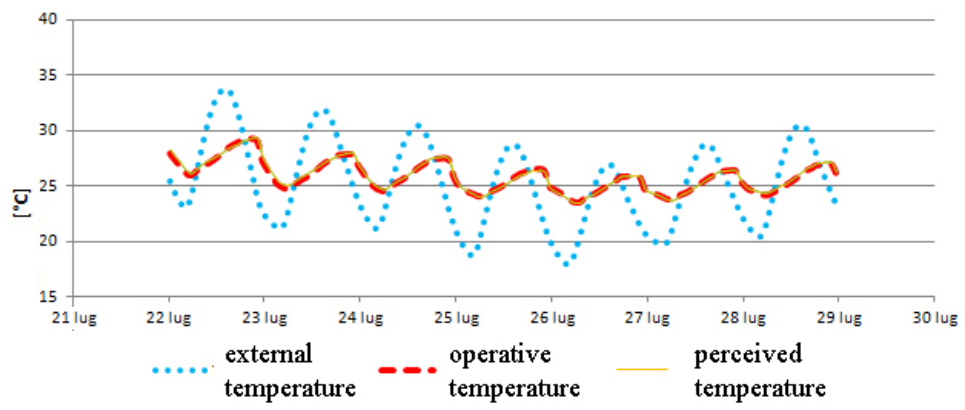

Figure 11: The operative temperature in the case of use of the envelope 4 combined with the natural night ventilation.

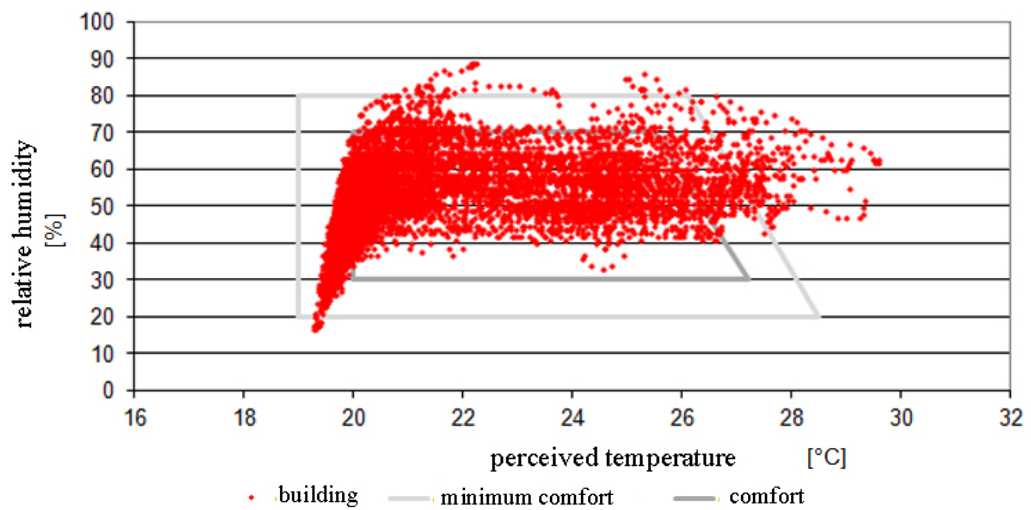

Figure 12: The analysis of the well-being of the users in B apartment in the case of use of the envelope 4 combined with the natural night ventilation. 
layers characterized by a high mass on the inner side (plasterboard made with PCM) and of passive cooling systems, guarantee the optimization of building performances during the summer, reducing the need to use air-conditioning systems.

\section{Conclusions}

The first applications of modular construction system based on polystyrene sheets have highlighted the difficulties associated with the importation of the systems studied in other climates and other cultural contexts.

The analyses and studies conducted in the early months of work propose some solutions, which aim to solve the main problems related to the winter behavior of envelopes made by polystyrene.

At a later stage the analysis of behavior during the summer of the construction system made by polystyrene sheets, showed that the best improvement is achievable through the simultaneous use of new envelope layers (as plasterboard made with PCM) and passive cooling systems (as the natural night ventilation).

Therefore is important to combine the correct design of building and its elements and the knowledge of users, explaining, in simple way, the rules to follow to enhance the performances of this kind of buildings.

As already mentioned, indeed, in order to reach the nearly-zero-energy building performances is required the combination of several factors that must be studied in a holistic manner.

\section{References}

[1] Directive 2010/31/EU of the European Parliament and of the Council of 19 May 2010 on the energy performance of buildings (recast).

[2] UNI/TS 11300 - 1:2008 - Prestazioni energetiche degli edifici. Parte 1.

[3] UNI/TS 11300 - 2:2008 - Prestazioni energetiche degli edifici. Parte 2.

[4] UNI/TS 11300 - 4:2012 - Prestazioni energetiche degli edifici. Parte 4.

[5] UNI EN ISO 10211:2008 - Ponti termici in edilizia. Flussi termici e temperature superficiali - Calcoli dettagliati.

[6] UNI EN ISO 13786:2008 - Prestazione termica dei componenti per edilizia Caratteristiche termiche dinamiche - Metodi di calcolo.

[7] Grosso, M., Il raffrescamento passivo degli edifici in zone a clima temperato, Maggioli Editore, Dogana, 2008.

[8] Di Perna, C., Stazi, F., Ursini Casalena, A., Stazi, A., "Massa e comfort: necessità di una adeguata capacità termica areica interna periodica", Costruire in laterizio, n. 126, pp. 52-59, 2008.

[9] Castell A., Medrano M., Castellón C., Cabeza Luisa F., Analysis of the simulation models for the use of pcm in buildings. Proc. of the 11th International Effstock 2009 - Thermal Energy Storage for Energy Efficiency and Sustainability. Stockholm, 2009. 